\title{
Anti-Tumor Activity and Safety of Multikinase Inhibitors in Advanced and/or Metastatic Thyroid Cancer: A Systematic Review and Network Meta-Analysis of Randomized Controlled Trials
}

Authors

Marina Tsoli ${ }^{*}$, Krystallenia I. Alexandraki ${ }^{~}{ }^{*}$, Maria-Eleni Spei ${ }^{*}$, Gregory A. Kaltsas ${ }^{1}$, Kosmas Daskalakis ${ }^{1,2}$

Affiliations

1 1st Department of Propaupedic Internal Medicine, National and Kapodistrian University of Athens, Athens, Greece

2 Department of Surgical Sciences, Uppsala University, Uppsala, Sweden

Key words

multikinase inhibitors, advanced/metastatic thyroid cancer, network meta-analysis

received 21.08.2019

accepted $\quad 01.10 .2019$

Bibliography

DOI https://doi.org/10.1055/a-1023-4214

Published online: 30.10 .2019

Horm Metab Res 2020; 52: 25-31

(c) Georg Thieme Verlag KG Stuttgart · New York ISSN 0018-5043

Correspondence

Dr. Kosmas Daskalakis

Endocrine Unit

1st Department of Propaupaedic Internal Medicine

Laiko University Hospital

National and Kapodistrian University of Athens

Mikras Asias 75

11527 Athens

Greece

Tel.: + 30698 8667113, Fax: + 302132061794

kosmas.das@gmail.com

Supplementary Material for this article is available online at http://www.thieme-connect.de/products.

\section{ABSTRACT}

Many trials have demonstrated prime antitumor activity of novel, small molecule multikinase inhibitors (MKIs) in advanced and/or metastatic thyroid cancer (TC). In this work, the PubMed, EMBASE, Cochrane Central Register of Controlled Trials, Web of Science, SCOPUS, and clinicaltrials.gov databases were searched. Quality/risk of bias were assessed using GRADE criteria. Randomized clinical trials (RCTs) comparing two or more systemic therapies in patients with advanced and/or metastatic thyroid cancer were assessed. A total of 1347 articles and 548 clinical trials in clinicaltrials.gov were screened. We included seven relevant RCTs comprising 1934 unique patients assigned to different MKIs. Two separate network meta-analyses included four RCTs in radioiodine refractory well-differentiated thyroid cancer (RR-WDTC) and three RCTs in medullary thyroid cancer (MTC), respectively; all with a low risk of bias. We identified three therapies for RR-WDTC: sorafenib [disease control rate (DCR) odds ratio (OR): 0.11 (95\% Cl: 0.03-0.40); progression-free survival (PFS) hazard ratio (HR): 1.99 (95\% Cl: 1.622.46)], vandetanib [DCR_OR:0.26 (95\% Cl: 0.06-1.24); PFS_ HR: 0.99 (95\% Cl: 0.82-1.20)] and lenvatinib [DCR_OR: 0.26 (95\% Cl: 0.05-1.33); PFS_HR: 0.99 (95\% Cl: 0.81-1.22)]; and the following therapies for MTC: vandetanib $300 \mathrm{mg}$ [objective response rate (ORR)_OR: 3.31 (95\% Cl: 0.68-16.22); vandetanib 150 mg ORR_OR: 0.60 (95\% Cl: 0.16-2.33)]; and cabozantinib [ORR_OR: 85.32 (95\% Cl: 5.22-1395.15)]. Serious side effect (SE) analysis per organ/system demonstrated a varying MKI SE profile across both RR-WDTC and MTC diagnoses, more commonly involving metabolic/nutritional disorders [OR: 2.07 [95\% Cl: 0.82-5.18)] and gastrointestinal SE [OR: 1.63 (95\% $\mathrm{Cl}: 1.0-2.66)]$. This network meta-analysis on advanced and/ or metastatic TC points towards a higher efficacy of lenvatinib in RR-WDTC. The included MKIs exhibit a varying SE profile across different organs/systems favoring a patient-tailored approach with the anticipated toxicities guiding clinicians' decisions.

* Denotes equal contribution 


\section{Introduction}

Although thyroid cancer (TC) constitutes the most common type of endocrine malignancy, it comprises a diverse range of histopathological entities the most common being well-differentiated thyroid cancer (WDTC). Generally, with the exception of anaplastic cancer, TC has been considered as having an overall good prognosis with most patients being cured by surgery although a subset with medullary thyroid cancer (MTC) may also exhibit an aggressive course. Traditional treatment for WDTC besides surgery includes radioactive iodine (RAI) and thyroid-stimulating hormone (TSH) suppression therapy, whereas surgery is the main treatment for MTC [1]. Nevertheless, some patients are diagnosed with established locally advanced and/or distant stage disease or even exhibit progress under the aforementioned standard therapies. The management of these patients is a multidisciplinary field with many recent innovations in TC genetics and molecular pathogenesis with pertinent therapeutic implications particularly in patients with MTC a subset of who may develop in the context of a familial syndrome [2]. Importantly, systemic cytotoxic chemotherapy has disappointing response rates in patients with unresectable and/or metastatic TC and also comes at high toxicity price [3]. However, recent randomized clinical trials (RCTs) have demonstrated prime anti-tumor activity of novel, small molecule multikinase inhibitors (MKIs) in TC, in particular RAI-refractory WDTC (RR-WDTC) and medullary TC (MTC), resulting in the approval of certain MKIs by the Food and Drug Administration (FDA).

Molecular alterations in the MAPK and the PI3k/Akt pathways have been recognized in the last few years as playing a pivotal role in gene expression linked with proliferation, cell migration and apoptosis inhibition of TC cells [2, 4, 5]. In WDTC, BRAFV600E constitutes a key mutation and is associated with aggressive histopathological features and dismal clinical outcomes [6, 7]. Other key mutations are the $\mathrm{H}-$, K-, and $\mathrm{N}$-RAS mutations found in follicular and poorly-differentiated TC; and PTEN deletions, also encountered in follicular TC $[2,8]$. In the MTC counterpart, most cases occur sporadically, whereas approximately $25 \%$ are linked with multiple endocrine neoplasia 2A (MEN2A) or MEN2B [9]. In particular, the majority of patients with sporadic form of MTC have somatic mutations identified in the gene encoding the RET protein, whereas patients with MEN2A or MEN2B exhibit germline RET mutations with close genotype-phenotype relation [10]. In sporadic MTC without RET mutations (35\%), RAS gene mutations are commonly encountered [11, 12]. Finally, the vascular endothelial growth factor (VEGF) and MET pathways are associated with angiogenesis, invasion, and promote metastasis in MTC $[13,14]$.

To date, a number of MKIs have been used in advanced and/or metastatic TC, including the MKI of VEGFRs 1-3, RET, RAF (including BRAFV600E), and PDGFR $\beta$, sorafenib [15]; the MKI of RET, VEGFR, and EGFR tyrosine kinases, vandetanib $[16,17]$; the MKI of VEGFRs 1-3, FGFR 1-4, PDGFR $\alpha$, RET, and KIT signaling networks, lenvatinib [18]; and the MKI of MET, VEGFR, and RET, cabozantinib [19]. However, translation of these results into clinical practice faces certain challenges, as a therapeutic reference standard for RR-WDTC and MTC in cases of advanced and/or metastatic disease is currently lacking and these MTTs have only been compared with placebo; hence, complicating clinical decision making in selecting one MKI agent over another.
Additionally, the RCTs in MKIs for RR-WDTC and MTC report treatment-related toxicities according to standard guidelines, that is, the National Cancer Institute Common Terminology Criteria for Adverse Events, and therefore constitute a complete resource of MKI-related toxicities. The present systematic review and network meta-analysis of MKIs for TC provides a comprehensive summary and a comparison of all the available randomized evidence on the antitumor activity and toxicities of novel MKI therapies in advanced and/or metastatic TC.

\section{Materials and Methods}

The present study was designed and conducted according to the Cochrane Guidelines for Systematic Reviews and Meta-analyses of Interventions and their extension for network meta-analysis [20-23].

\section{Search strategy and study selection}

Our aim was to identify all potentially eligible RCTs comparing systemic MKIs in advanced and/or metastatic TC. A broad search algorithm using MeSH terms and text words in the abstract in combination with a therapeutic intervention and a study design filter was developed. Search strategy and the applied filters regarding treatment selection and study design are presented in the Supplement (Supplementary $>$ Table 1S). The PubMed, Embase, SCOPUS, Web of Science and the Cochrane Central Register of Controlled Trials were searched through until March 25, 2019. No language or date restrictions were applied. The website of ClinicalTrials.gov for potentially eligible unpublished trials was also searched through. Key search terms included thyroid cancer, therapy, and randomized controlled trial. We included RCTs comparing a MKI with placebo or different doses of the same agent reporting disease control rate, objective response rate, progression-free survival and/or side-effect incidence. Two of the authors (MT and KD) worked in duplicate independently and screened all potentially eligible titles and abstracts, as well as the full-text manuscripts of all potentially relevant trials to finalize eligibility. Disagreements were resolved by consensus between MT and KD or discussed with a third author (GK). Preferred Reporting Items for Systematic Reviews and Meta-Analyses (PRISMA) guidelines for reporting were followed [21-23]. A study protocol for this meta-analysis was not published or registered before the study was undertaken.

\section{Outcomes and data extraction}

The primary outcomes with regards to MKIs' antitumor activity were disease control rate (DCR), objective response rate (ORR) and progression-free survival (PFS). Secondary outcomes were MKIs' safety profile and included serious side effects (SE). Absolute values of DCR, ORR and SE; hazard ratios (HR) with $95 \%$ Confidence Intervals (CI) for PFS rates were extracted. Data on tumor type, study size and industry sponsorship were also extracted. Two of the authors (MT and KD) extracted all data in duplicate and independently. As in study selection, discordances in data extraction were resolved by consensus. 


\section{Risk of bias and quality of evidence assessment}

The risk of bias for all included studies was assessed with the Grading of Recommendations Assessment, Development and Evaluation (GRADE) using the Cochrane Risk of Bias Tool [24, 25]. Scores were given for the following standard domains: random sequence generation, allocation concealment, blinding of participants and personnel, blinding of outcome assessment, completeness of outcome data, for each domain. Two of the authors (MT and KD) assessed all RCTs in duplicate and independently. Disagreements on risk of bias assessments were resolved by consensus (between MT and KD) and/or discussed with a third reviewer (GK).

\section{Statistical analysis}

We conducted a network meta-analysis with a frequentist approach. The investigated end-points included DCR, ORR, PFS and serious SE (overall and divided by organ/system) for RR-WDTC and MTC. We applied a continuity correction for studies with a 0 cell count by adding 0.5 to all cell frequencies [26]. We assessed heterogeneity by the between study-variance $\mathrm{T}$, Cochran $\mathrm{Q}$ and $\mathrm{I}^{2}$. We assessed inconsistency by the global inconsistency test and implemented the inconsistency model in which direct and indirect estimates were compared and a calculation of the between-design part of Cochran $\mathrm{Q}$ analysis. Having fit the consistency and inconsistency model, we produced network forest plots to evaluate the effect size of each RCT and each treatment. However, neither consistency nor heterogeneity for all the investigated networks could be confidently determined; hence, consistency and lack for heterogeneity was assumed for all networks in the study.

Interval forest plots were used with combined effect estimates (i. e., OR and HRs with $95 \% \mathrm{Cls}$ and size of boxes proportional to the inverse of the SEs). We ranked therapies using the surface under the cumulative ranking (SCURA) command in STATA to identify superiority among the investigated treatments. The data were presented with ranking plots and clustered ranking plots (DCR-PFS plot) of competing therapies for RR-WDTC and MTC separately, as appropriate. We stratified the meta-analysis by subgroups of MKI serious SE profile across different organ/systems. We used a random-effects model to present study- specific odds ratios (OR) [27]. To explore heterogeneity between the studies the $\mathrm{I}^{2}$ statistics were used [28]. When $\mathrm{I}^{2}$ was $>0.50 \%$ the statistical heterogeneity was considered substantial. The level of statistical significance was set at $5 \%$ (twotailed $\mathrm{p}<0.05)$. We used the mvmeta application in the STATA package (version 13.1; StataCorp, College Station, TX, USA) [29, 30].

\section{Results}

\section{Study selection and risk of bias assessment}

We initially screened 1347 titles and abstracts from all databases and additional 548 clinical trials in clinicaltrials.gov and identified 25 potentially eligible RCT reports ( nally, a total of seven RCTs reported DCR, ORR and/or PFS and were included in the network meta-analyses. Some of the RCTs were reported in more than one publication, whereas the results of two RCTs were solely available from clinicaltrials.gov, that is, not yet published. Only patients with locally advanced and/or metastatic TC were included. In particular, four RCTs included RR-WDTC and three included MTC. A total of 1934 unique patients were recruited; four different MKIs were evaluated. All RCTs in the network meta-analysis were industry sponsored. RCT characteristics are provided in $>$ Supplementary Table 25 .

Among seven included RCTs, high risk for bias in random sequence generation (selection bias), allocation concealment (selection bias), blinding participants and personnel (performance bias), blinding the outcome assessment (detection bias), incomplete outcome data (attrition bias), and selective reporting (reporting bias) was evident in none of the studies ( $\triangleright$ Supplementary Table 3S).

\section{Antitumor activity in RR-WDTC}

Four RCTs compared DCR and PFS for three different MKIs in RRWDTC ( $\triangleright$ Supplementary Fig. 2S a). The network meta-analysis found that all MKI monotherapies studied were highly effective compared to placebo both in terms of DCR and PFS analysis. The corresponding figures ( $\triangleright$ Fig. 1a, b) present the estimated summary effects for all comparisons of DCR and PFS, respectively. Specifically, sorafenib exhibited an OR with regards to DCR of 0.11 [95\% Cl: 0.03-0.40 and a PFS_HR of 1.99 (95\% Cl: 1.62-2.46)]; for vandetanib $300 \mathrm{mg}$ the corresponding pooled estimates were DCR_ OR: 0.26 (95\% Cl: 0.06-1.24) and PFS_HR: 0.99 (95\% Cl: $0.82-$ 1.20); and for lenvatinib, DCR_OR was 0.26 (95\% Cl: 0.05-1.33) and PFS_HR was 0.99 (95\% Cl: $0.81-1.22)$, respectively ( $\bullet$ Fig. 1a, b). A clusterrank plot of PFS and DCR is given in $>$ Fig. 1c. The quality of evidence in RR-WDTC was high for all the included studies.

\section{Antitumor activity in MTC}

Three RCTs assessed ORR for two different therapies in MTC ( Supplementary Fig. S2 b). One RCT for MTC in the network meta-analysis did not report DCR, but only ORR; hence, ORR network analysis was undertaken. The network meta-analysis demonstrated that cabozantinib with regards to ORR analysis was highly effective. Cabozantinib resulted in the highest ORR [OR: 85.32 (95\% Cl: 5.22-1395.15)] followed by vandetanib $300 \mathrm{mg}$ [ORR_OR: 3.31 (95\% Cl, 0.68-16.22)] and vandetanib $150 \mathrm{mg}$ [ORR_OR: 0.60 [95\% Cl, 0.16-2.33)] (• Fig. 2a, b).

Two RCTs only assessed PFS for two different therapies (cabozantinib and vandetanib $300 \mathrm{mg}$ ) in MTC. The lowest hazard for progression was found after treatment with cabozantinib treatment [HR: 0.28 (95\% Cl, 0.19-0.40)], followed by vandetanib $300 \mathrm{mg}$ [HR: $0.46(95 \% \mathrm{Cl}, 0.31-0.69)]$. Both therapies significantly reduced the hazard for progression compared with placebo. The quality of evidence in MTC was high for all the included studies.

\section{Serious toxicities profile}

Four RCTs compared serious SE for three different MKIs in RR-WDTC ( Fig. 3a). Sorafenib exhibited an OR for serious SE of 0.30 (95\% Cl: 0.12-0.72); for vandetanib $300 \mathrm{mg}$ SE_OR was 0.49 (95\% Cl: 0.15-1.68); and for lenvatinib SE_OR was 0.96 (95\% Cl: 0.31-3.01), respectively ( $\triangleright$ Fig. $\mathbf{3 a}$ ). For MTC, the summary effect estimates were SE_OR: 1.20 (95\% Cl, 0.45-3.19) for vandetanib $300 \mathrm{mg}$, SE_ OR: 1.07 (95\% Cl, 0.23-4.92) for vandetanib $150 \mathrm{mg}$, and SE_OR: 0.41 (95\% Cl: 0.22-0.75) for cabozantinib, as compared to placebo ( $\vee$ Fig. 3b). We conducted a subgroup meta-analysis per organ/ system in serious SE. Our findings showed a varying MKI SE profile 
a

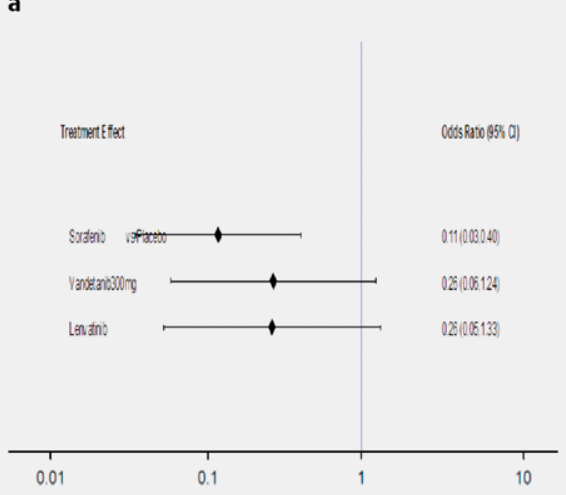

b

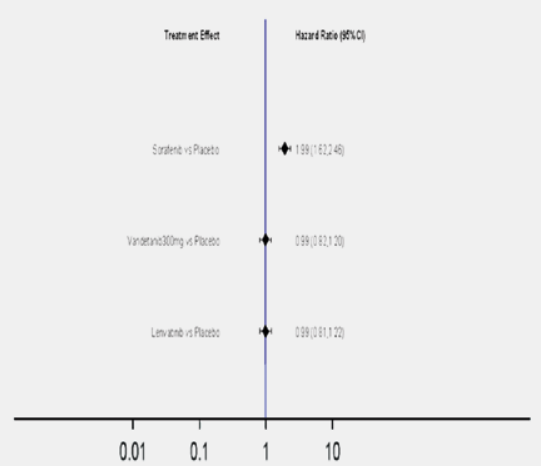

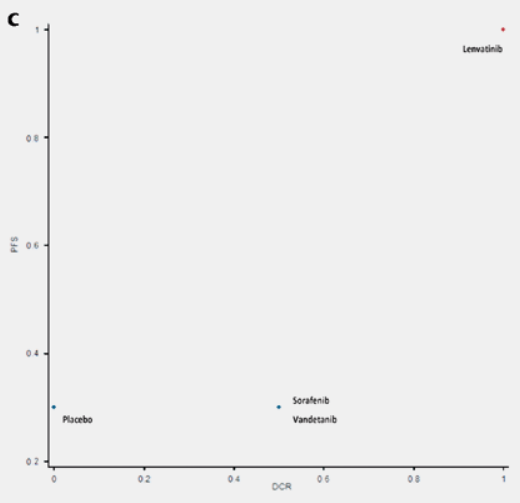

- Fig. 1 a Interval plot of Disease Control Rate (DCR; Odds ratios with 95\% Confidence Intervals); b Interval plot of Progression-free Survival (PFS; Hazard ratios with $95 \%$ Confidence Intervals); and $\mathbf{c}$ respective clusterrank plot of PFS and DCR in radioiodine refractory well-differentiated thyroid cancer.
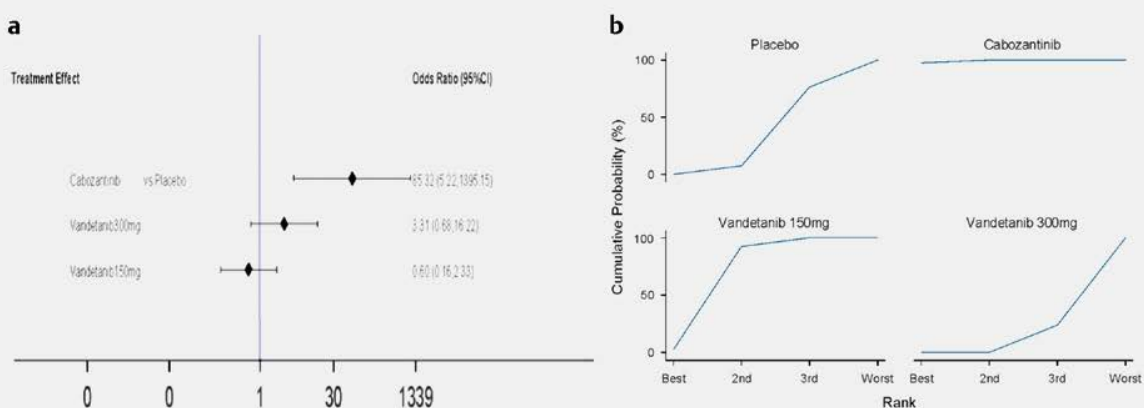

- Fig. 2 a Interval plot of Objective Response Rate (ORR; Odds ratios with 95\% Confidence Intervals) and b Rankogram of estimated probabilities of each treatment being the best based on ORR in Medullary Thyroid Cancer.

a

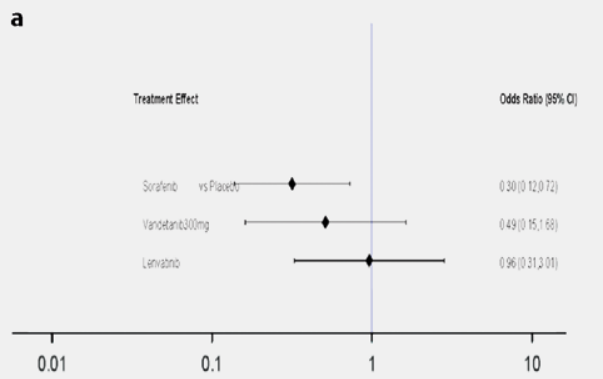

b

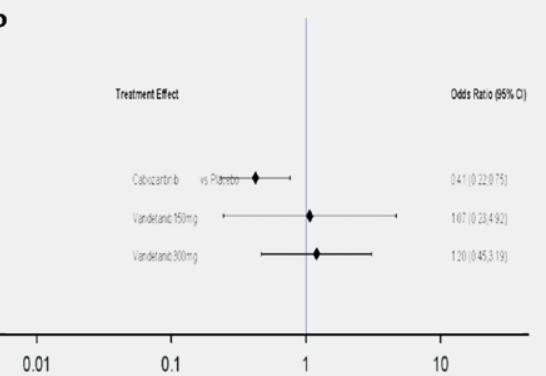

- Fig. 3 a Multikinase Inhibitor Serious Toxicities risk assessment (odds ratio with 95\% Confidence Intervals) in Radioiodine Refractory Well-Differentiated Thyroid Cancer and b Mulitikinase Inhibitor Serious Toxicities risk assessment (odds ratio with $95 \%$ Confidence Intervals) in Medullary Thyroid Cancer.

across both RR-WDTC and MTC diagnoses, more commonly involving metabolic/nutritional disorders (OR: 2.07, $95 \% \mathrm{Cl}$ : 0.82-5.18) and gastrointestinal SE (OR: 1.63, 95\% Cl:1.00-2.66) (๖ Fig. 4).

\section{Representation in international guidelines}

Among the five published RCTs included in the present meta-analysis, four RCTs were included in the latest American Thyroid Association (ATA) consensus guidelines for WDTC and MTC and two RCTs in 


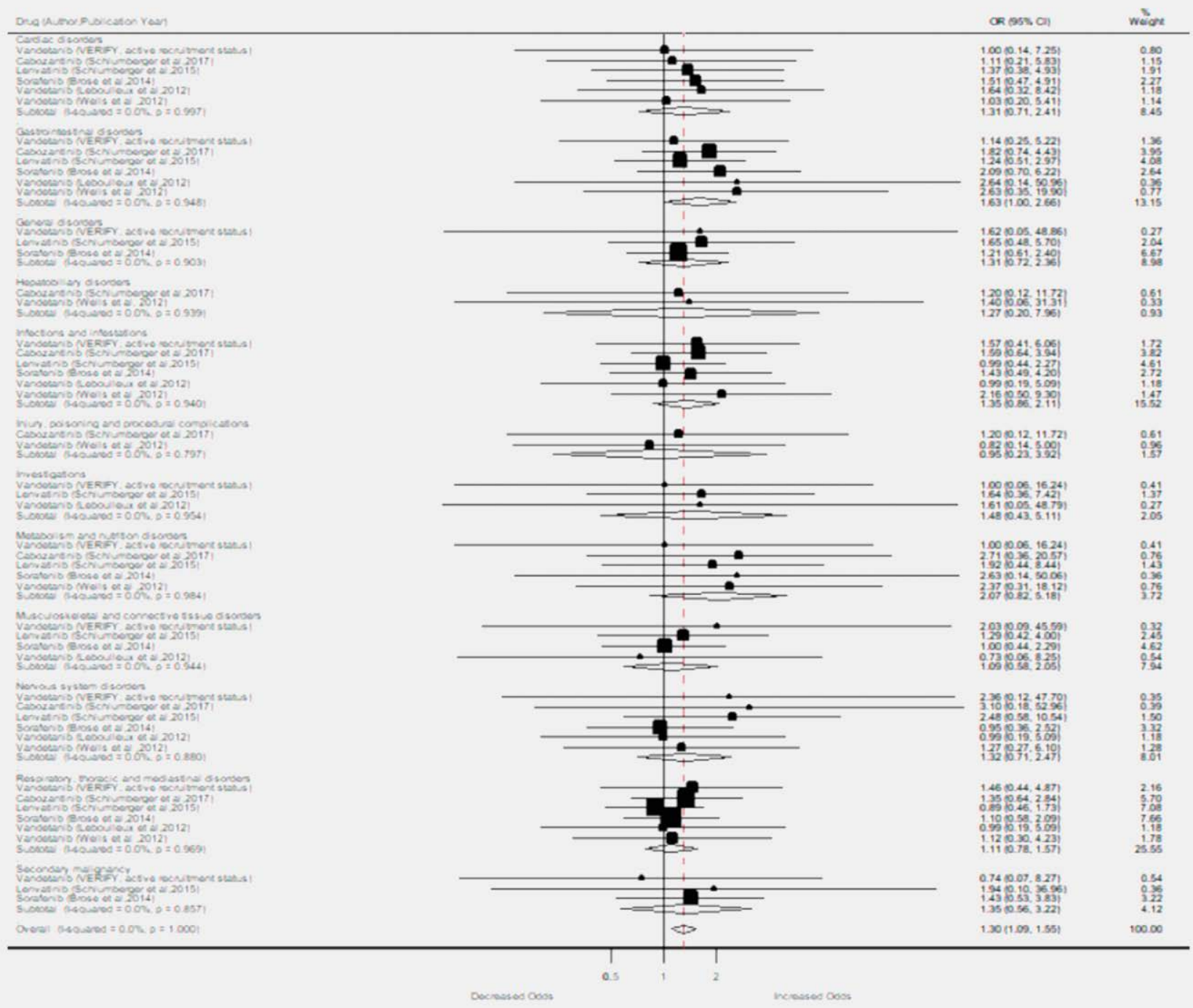

- Fig. 4 Multikinase Inhibitors Serious Toxicities profile per organ/system in patients with Advanced and/or Metastatic Thyroid Cancer (Odds Ratios with $95 \%$ Confidence Intervals).

the European Thyroid Association (ETA) guidelines for MTC [31-33]. However, the ETA guidelines for WDTC are from 2008; hence, MKIs for RR-WDTC are not considered in these guidelines and an ETA recommendation is still awaited.

\section{Discussion}

Herein, we present a systematic review and network meta-analysis of available RCTs evaluating the antitumor activity and safety profile of MKI therapies for advanced and/or metastatic TC. We identified seven RCTs that randomized 1934 patients with advanced and/or metastatic TC to four different MKI therapies. Our results suggest a range of $\mathrm{MKI}$ monotherapies that are superior to placebo, including sorafenib, vandetanib $(300 \mathrm{mg})$, and lenvatinib in RR-WDTC, and vandetanib and cabozantinib in MTC. Our findings point towards a higher efficacy among the investigated MKIs with regards to antitumor activity of lenvatinib in RR-WDTC. In ad- dition, MKI exhibit a broad range of risk for serious SE with regards to each drug's safety profile with varying treatment-related SE across different organs/systems; hence, favoring a more patient-tailored approach with the anticipated toxicities guiding clinicians' therapeutic decisions. In particular, serious SE across both RR-WDTC and MTC diagnoses, showed that there is evidence of a profile more commonly involving metabolic/nutritional disorders and gastrointestinal SE. Our results also highlight the need for further research in assessing serious toxicities and effects on quality of life for different MKI therapies.

In MTC, the ZETA trial on vandetanib $(300 \mathrm{mg}$ ) reported an ORR as high as 45 vs. $8 \%$ in the placebo group and also a PFS benefit with a median of 30.5 vs. 19.3 months in the placebo group (HR 0.46, $95 \% \mathrm{Cl} 0.31-0.69, \mathrm{p}<0.001)$. On the contrary, in the EXAM trial on cabozantinib, participants needed radiological evidence of disease progression to be eligible. The study reported an ORR of 28 vs. $0 \%$ in the placebo group and demonstrated a PFS prolongation 
of 11.2 vs. 4.0 months in the placebo group (HR: $0.28,95 \% \mathrm{Cl} 0.19-$ $0.40 ; p<0.001$. With regards to each agent's safety profile, unlike vandetanib, cabozantinib was not linked with QTc prolongation. However, even if the PFS for cabozantinib appears to be considerably shorter than that of vandetanib, the EXAM and ZETA populations may not be directly comparable, due to between study differences with respect to eligibility criteria, i. e. a MTC population with progressive disease in the EXAM trial. Hence, safe conclusions cannot be derived regarding which agent is superior and the results of our ORR network meta-analysis in the setting of MTC have to be interpreted with caution. Therapeutic decisions regarding MKI selection in MTC should probably be based on patient general status and comorbidities with particular focus on the anticipated toxicity profiles across different organ/systems.

The GRADE system was applied to assess the risk of bias of the included RCTs and the confidence in effect estimates for all comparisons. Importantly, the end points assessed in this network meta-analysis were DCR, ORR and/or PFS, instead of overall survival (OS), which is the most relevant clinical end point. However to date, no clinical trial has demonstrated an OS benefit from the use of any MKIs in advanced and/or metastatic TC, although this is likely to be due to the high rates of crossover in the placebo groups of the included RCTs and the data immaturity. Additionally, MKIs have been linked with a wide range of toxicities that have an impact on the patients' quality of life. Determining the right time and choice of agent to initiate the MKI treatment represents one of the most important future tasks, as for example the radioiodine refractory feature per se is not sufficient to determine if a WDTC patient is a good candidate for MKI therapy and the currently available biomarkers, that is, thyroglobulin and calcitonin lack a predictive value with regards to treatment selection and monitoring response to MKIs. In addition, the clinical effects of the investigated MKIs in WDTC have not been clearly linked with mutation status, e. g. BRAF or RAS mutations for patients treated with lenvatinib. On the other hand for the MTC counterpart, exploratory assessment of ORR and PFS in the EXAM trial exhibited a larger treatment effect of cabozantinib in patients with RET M918T mutation-positive tumors [19]. Finally, it remains to be determined the exact sequencing of lines of treatments upon disease progression since there are many treatments not yet tested in RCTs, including radioiodine resensitization, immunotherapy, novel inhibitors of specific molecular targets as tyrosine or MEK kinases as well as checkpoint factors [34].

Patients who are candidates for MKIs should be thoroughly counseled on the potential risks and benefits of this particular therapy, as these agents are associated with many SE including fatigue, hypertension, hepatotoxicity, skin changes and numerous gastroenterological disorders. These potential SE have a certain probability of negatively impacting quality of life and necessitating dosage reductions or treatment discontinuation. Nevertheless, MKIs are linked with more severe risks including thrombosis, bleeding, heart failure, hepatotoxicity, gastrointestinal tract fistula formation, and intestinal perforation [35]. In the present meta-analysis, serious SEs network analysis for RR-WDTC exhibited a higher risk in patients treated with lenvatinib, whereas in MTC, vandetanib at a dose of $300 \mathrm{mg}$ was associated with a higher risk for serious SE. Further analysis per organ/system demonstrated a varying MKI SE profile for advanced and/or metastatic TC, commonly involving metabolic/nutritional disorders and gastrointestinal SE; hence, discouraging MKI therapy in patients with certain comorbidities, e. g. active or recent intestinal disease, including recent Gl bleeding, diverticulitis, inflammatory bowel disease, recent bowel resection as per 2015 ATA guidelines [31].

This study has some limitations. Most RCTs had an unclear risk of bias due to lack of reporting details on random sequence generation and allocation concealment. Due to the very low number of RCTs included in the analysis, the assessment of inconsistency, as well as heterogeneity and publication bias was limited. Nevertheless, the low number of RCTs may have introduced imprecision to the network comparisons, namely wide $95 \%$ Cls may indeed include statistically significant, yet clinically irrelevant effects. Finally, our study is subject to biases or confounders encountered in the original RCTs; hence, the findings are generalizable only to patient groups eligible for these trials. However, the strengths of our study were that we applied a comprehensive search strategy with a sensitive search algorithm, obtaining data also from unpublished RCTs targeting all available randomized evidence. We included studies reporting DCR, ORR and/or PFS in patients with RR-WDTC and MTC separately; thus ensuring directness. Additionally, these well-defined TC populations and patient outcomes resulted in a network with rather high transitivity. Nevertheless, a comprehensive analysis of serious treatment-related SE reported in RCTs on MKIs is critical, as it may constitute a reference for clinicians treating TC.

Our systematic review and network meta-analysis have implications for clinicians, researchers and guideline committees. It provides a comprehensive overview of the randomized evidence on MKI therapies for advanced and/or metastatic TC as well as the best possible comparison of therapies that have not been directly compared in RCTs with the aims to assist clinical decision making and guide further research in the field. MKI antitumor activity results, and serious toxicities profile, as presented in our network analysis, but also across different organ/systems may aid in therapeutic decisions and also facilitate the implementation of surveillance strategies for MKI-related toxicities. In the era of personalized medicine, validated predictive biomarkers including histopathological and molecular parameters with the potential to guide MKI treatment selection at the individual level are warranted. Toxicities encountered in MKI treatment prompt further investigation with particular focus on quality of life aspects in TC patients to achieve a balance between antitumor activity and toxicities.

\section{Conflict of Interest}

The authors declare that they have no conflict of interest.

References

[1] Cabanillas ME, McFadden DG, Durante C. Thyroid cancer. Lancet 2016; 388: 2783-2795

[2] Bhaijee F, Nikiforov YE. Molecular analysis of thyroid tumors. Endocr Pathol 2011; 22: 126-133

[3] Sherman SI. Early clinical studies of novel therapies for thyroid cancers. Endocrinol Metab Clin North Am 2008; 37: 511-524 xi 
[4] Abubaker J, Jehan Z, Bavi P et al. Clinicopathological analysis of papillary thyroid cancer with PIK3CA alterations in a Middle Eastern population. J Clin Endocrinol Metab 2008; 93: 611-618

[5] Xing M. Molecular pathogenesis and mechanisms of thyroid cancer. Nat Rev Cancer 2013; 13: 184-199

[6] Elisei R, Ugolini C, Viola D et al. BRAF(V600E) mutation and outcome of patients with papillary thyroid carcinoma: A 15-year median follow-up study. J Clin Endocrinol Metab 2008; 93: 3943-3949

[7] Xing M, Alzahrani AS, Carson KA et al. Association between BRAF V600E mutation and mortality in patients with papillary thyroid cancer. JAMA 2013; 309: 1493-1501

[8] Romei C, Fugazzola L, Puxeddu E et al. Modifications in the papillary thyroid cancer gene profile over the last 15 years. J Clin Endocrinol Metab 2012; 97: E1758-E1765

[9] Grubbs EG, Ng PK, Bui ] et al. RET fusion as a novel driver of medullary thyroid carcinoma. J Clin Endocrinol Metab 2015; 100: 788-793

[10] Eng C, Clayton D, Schuffenecker I et al. The relationship between specific RET proto-oncogene mutations and disease phenotype in multiple endocrine neoplasia type 2 . International RET mutation consortium analysis. JAMA 1996; 276: 1575-1579

[11] Ji JH, Oh YL, Hong M et al. Identification of driving ALK fusion genes and genomic landscape of medullary thyroid cancer. PLoS Genet 2015; 11: e1005467

[12] Boichard A, Croux L, Al Ghuzlan A et al. Somatic RAS mutations occur in a large proportion of sporadic RET-negative medullary thyroid carcinomas and extend to a previously unidentified exon. J Clin Endocrinol Metab 2012; 97: E2031-E2035

[13] Papotti M, Olivero M, Volante M et al. Expression of Hepatocyte Growth Factor (HGF) and its Receptor (MET) in Medullary Carcinoma of the Thyroid. Endocr Pathol 2000; 11: 19-30

[14] Soh EY, Duh QY, Sobhi SA et al. Vascular endothelial growth factor expression is higher in differentiated thyroid cancer than in normal or benign thyroid. J Clin Endocrinol Metab 1997; 82: 3741-3747

[15] Brose MS, Nutting CM, Jarzab B et al. Sorafenib in radioactive iodine-refractory, locally advanced or metastatic differentiated thyroid cancer: a randomised, double-blind, phase 3 trial. Lancet 2014; 384: 319-328

[16] Leboulleux S, Bastholt L, Krause T et al. Vandetanib in locally advanced or metastatic differentiated thyroid cancer: a randomised, double-blind, phase 2 trial. Lancet Oncol 2012; 13: 897-905

[17] Wells SA Jr, Robinson BG, Gagel RF et al. Vandetanib in patients with locally advanced or metastatic medullary thyroid cancer: A randomized, double-blind phase III trial. J Clin Oncol 2012; 30: 134-141

[18] Schlumberger M, Tahara M, Wirth L] et al. Lenvatinib versus placebo in radioiodine-refractory thyroid cancer. N Engl J Med 2015; 372: $621-630$

[19] Schlumberger M, Elisei R, Muller S et al. Overall survival analysis of EXAM, a phase III trial of cabozantinib in patients with radiographically progressive medullary thyroid carcinoma. Ann Oncol 2017; 28: 2813-2819
[20] Higgins JPTGS. Cochrane Handbook for Systematic Reviews of Interventions. In: The Cochrane Collaboration. 2019

[21] Moher D, Liberati A, Tetzlaff J et al. Preferred reporting items for systematic reviews and meta-analyses: The PRISMA statement. Ann Intern Med 2009; 151: 264-269 W264

[22] Liberati A, Altman DG, Tetzlaff ] et al. The PRISMA statement for reporting systematic reviews and meta-analyses of studies that evaluate health care interventions: Explanation and elaboration. Ann Intern Med 2009; 151: W65-W94

[23] Hutton B, Salanti G, Caldwell DM et al. The PRISMA extension statement for reporting of systematic reviews incorporating network meta-analyses of health care interventions: Checklist and explanations. Ann Intern Med 2015; 162: 777-784

[24] Puhan MA, Schunemann HJ, Murad MH et al. A GRADE Working Group approach for rating the quality of treatment effect estimates from network meta-analysis. BMJ 2014; 349: g5630

[25] Brignardello-Petersen R, Bonner A, Alexander PE et al. Advances in the GRADE approach to rate the certainty in estimates from a network meta-analysis. J Clin Epidemiol 2018; 93: 36-44

[26] Sweeting MJ, Sutton AJ, Lambert PC. What to add to nothing? Use and avoidance of continuity corrections in meta-analysis of sparse data. Stat Med 2004; 23: 1351-1375

[27] Borenstein M, Hedges LV, Higgins JP et al. A basic introduction to fixed-effect and random-effects models for meta-analysis. Res Synth Methods 2010; 1: 97-111

[28] Higgins JP, Thompson SG. Quantifying heterogeneity in a meta-analysis. Stat Med 2002; 21: 1539-1558

[29] Chaimani A, Higgins JP, Mavridis D et al. Graphical tools for network meta-analysis in STATA. PLoS One 2013; 8: e76654

[30] White IR. 'Network Meta-analysis'. Stata ] 2015; 15: 951-985

[31] Haugen BR, Alexander EK, Bible KC et al. 2015 American Thyroid Association Management guidelines for adult patients with thyroid nodules and differentiated thyroid cancer: The American Thyroid Association guidelines task force on thyroid nodules and differentiated thyroid cancer. Thyroid 2016; 26:: 1-133

[32] Wells SA Jr., Asa SL, Dralle $\mathrm{H}$ et al. American Thyroid Association guidelines task force on medullary Thyroid C. Revised American Thyroid Association guidelines for the management of medullary thyroid carcinoma. Thyroid 2015; 25: 567-610

[33] Schlumberger M, Bastholt L, Dralle $\mathrm{H}$ et al. European Thyroid Association Task F. 2012 European thyroid association guidelines for metastatic medullary thyroid cancer. Eur Thyroid J 2012; 1: 5-14

[34] Tsoukalas N, Tsapakidis K, Alexandraki KI. The role of palbociclib in thyroid carcinoma with BRAF mutation. Gland Surg 2018; 7: S82-S85

[35] Schutz FA, Je Y, Richards C], Choueiri TK. Meta-analysis of randomized controlled trials for the incidence and risk of treatment-related mortality in patients with cancer treated with vascular endothelial growth factor tyrosine kinase inhibitors. J Clin Oncol 2012; 30: 871-877 


\section{Supplementary Material}

\section{- Supplementary Table 15 Search strategy.}

\section{Databases assessed}

PubMed, Embase, Cochrane Central Register of Controlled Trials, SCOPUS, Web of Science and Trial registry through clinicaltrials.gov

\section{Search Strategy}

("cancer of the thyroid" [Mesh] OR "cancer of thyroid"[Mesh] OR "cancer, thyroid"[Mesh] OR

“carcinoma, thyroid” [Mesh] OR “Carcinoma, Medullary”[Mesh] OR “Carcinoma, papillary, follicular”[Mesh] OR “Thyroid Cancer, Papillary”[Mesh] OR "Thyroid Carcinoma, Anaplastic"[Mesh] OR "Anaplastic Thyroid Cancer”[Mesh] OR “Thyroid Cancer, Follicular”[Mesh])

\section{Therapy Search Filter}

therapy[sh] OR “diet therapy"[sh] OR "drug therapy"[sh] OR radiotherapy[sh] OR surgery[sh] OR resection OR debulk* OR cryoablat * OR cryosurger * OR radioablat * OR radioactive iodine ablation OR radioactive iodine * OR radioiodine * OR RAI * OR OR I131 * OR iodine-I131 * OR lymph node dissection OR neck dissection OR central neck dissection OR lateral neck dissection OR chemotherapy OR ethanol injection OR chemotherapies OR radiotherapy OR external beam radiotherapy OR targeted molecular therapy OR radiopeptide OR recombinant human TSH OR rhTSH OR Axitinib OR Motesanib diphosphate OR Pazopanib OR Sorafenib OR Sunitinib OR Gefitinib OR Vandetanib OR Lenvatinib OR Cabozantinib OR Everolimus OR Thalidomide OR Lenalidomide

\section{Study design Filter}

randomized controlled trial[pt] OR controlled clinical trial[pt] OR randomized[tiab] OR placebo[tiab] OR "drug therapy"[sh] OR randomly[tiab] OR trial[tiab] OR groups[tiab]) NOT (“animals”[mh] NOT (“humans”[mh] AND “animals”[mh]).
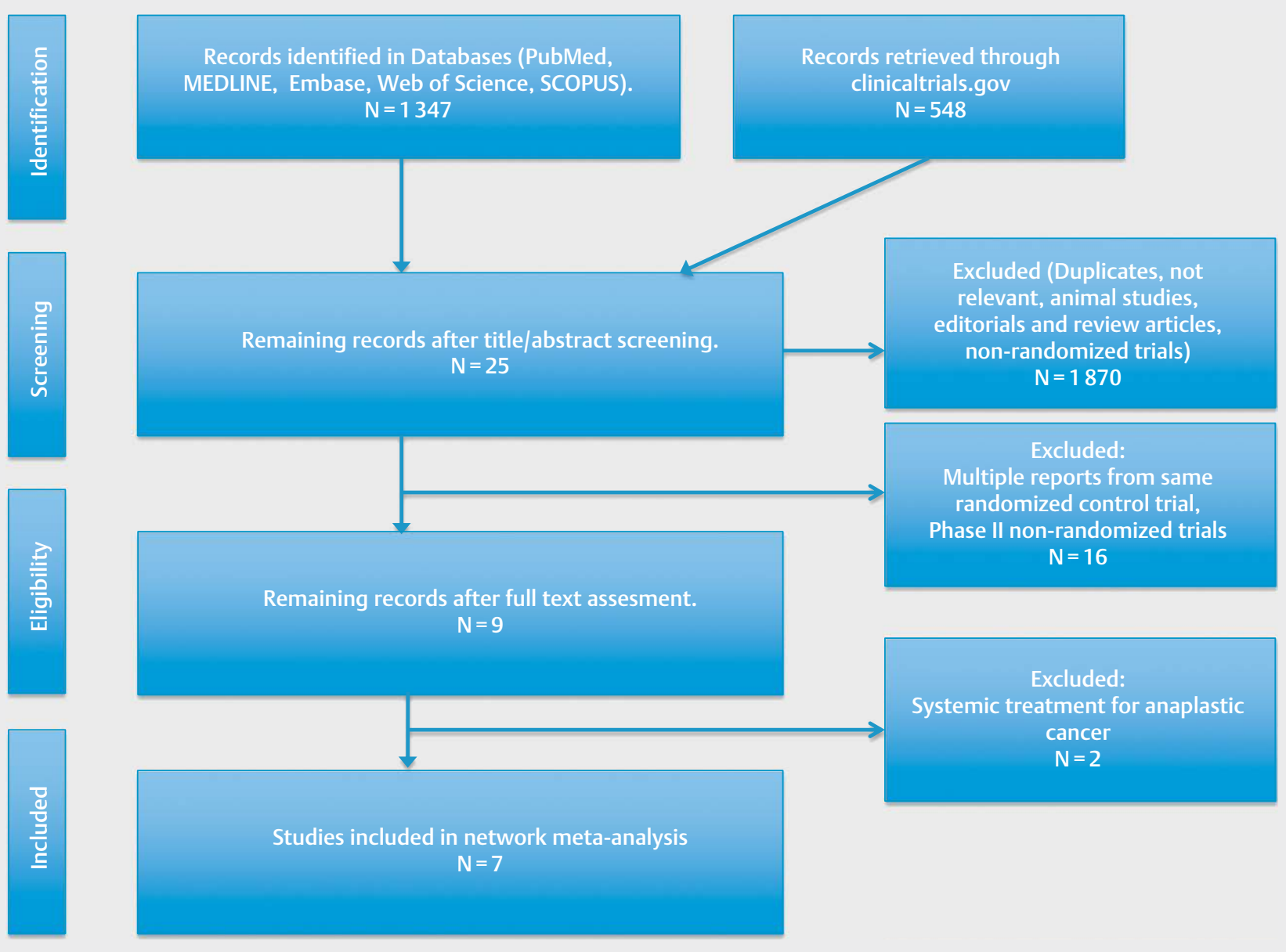

- Supplementary Figure 1S PRISMA flowchart of search results. 


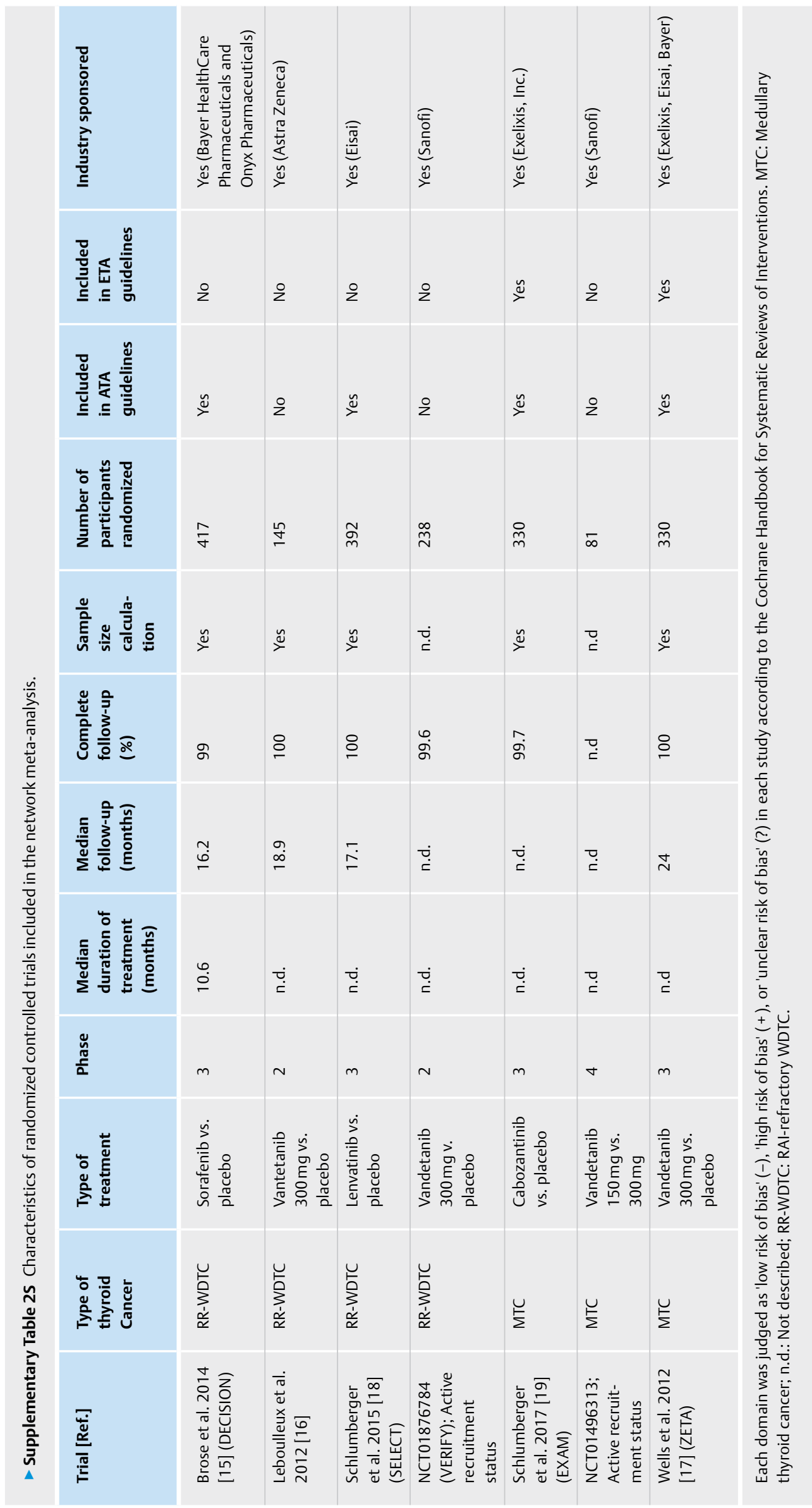


- Supplementary Table 35 Risk of bias summary: Authors' judgments about each risk of bias item for each included study.

\begin{tabular}{|c|c|c|c|c|c|c|c|c|}
\hline Study [Ref.] & $\begin{array}{l}\text { Comparison } \\
\text { groups }\end{array}$ & $\begin{array}{l}\text { Random } \\
\text { sequence } \\
\text { generation }\end{array}$ & $\begin{array}{l}\text { Allocation } \\
\text { conceal- } \\
\text { ment }\end{array}$ & $\begin{array}{l}\text { Blinding of } \\
\text { participants } \\
\text { and } \\
\text { personnel }\end{array}$ & $\begin{array}{l}\text { Blinding of } \\
\text { outcome } \\
\text { assessment }\end{array}$ & $\begin{array}{l}\text { Incomplete } \\
\text { outcome } \\
\text { data }\end{array}$ & $\begin{array}{l}\text { Selective } \\
\text { reporting }\end{array}$ & $\begin{array}{l}\text { Other } \\
\text { bias }\end{array}$ \\
\hline \multicolumn{9}{|l|}{ RR-WDTC } \\
\hline Brose et al. 2014 [15] & $\begin{array}{l}\text { Sorafenib vs. } \\
\text { placebo }\end{array}$ & $?$ & $?$ & $(-)$ & $(-)$ & $(-)$ & $(-)$ & $?$ \\
\hline $\begin{array}{l}\text { Leboulleux et al. } 2012 \\
\text { [16] }\end{array}$ & $\begin{array}{l}\text { Vantetanib } \\
300 \mathrm{mg} \text { vs. } \\
\text { placebo }\end{array}$ & $(-)$ & $?$ & $(-)$ & $(-)$ & $(-)$ & $(-)$ & $?$ \\
\hline $\begin{array}{l}\text { Schlumberger et al. } \\
2015 \text { [18] }\end{array}$ & $\begin{array}{l}\text { Lenvatinib vs. } \\
\text { placebo }\end{array}$ & $(-)$ & $?$ & $(-)$ & $(-)$ & $(-)$ & $(-)$ & $?$ \\
\hline $\begin{array}{l}\text { NCT01876784 } \\
\text { (VERIFY); Active } \\
\text { recruitment status }\end{array}$ & $\begin{array}{l}\text { Vandetanib } \\
300 \mathrm{mg} \text { vs. } \\
\text { placebo }\end{array}$ & $?$ & $?$ & $(-)$ & $(-)$ & $(+)$ & $(-)$ & $?$ \\
\hline \multicolumn{9}{|l|}{ MTC } \\
\hline $\begin{array}{l}\text { Schlumberger et al. } \\
2017 \text { [19] }\end{array}$ & $\begin{array}{l}\text { Cabozantinib } \\
\text { vs. placebo }\end{array}$ & $?$ & $?$ & $(-)$ & $(-)$ & $(-)$ & $(-)$ & $?$ \\
\hline $\begin{array}{l}\text { NCT01496313; Active } \\
\text { recruitment status }\end{array}$ & $\begin{array}{l}\text { Vandetanib } \\
150 \mathrm{mg} \text { vs. } \\
300 \mathrm{mg}\end{array}$ & $?$ & $?$ & $(-)$ & $(-)$ & $(+)$ & $(-)$ & $?$ \\
\hline $\begin{array}{l}\text { Wells et al. } 2012 \text { [17] } \\
\text { (ZETA) }\end{array}$ & $\begin{array}{l}\text { Vandetanib } \\
300 \mathrm{mg} \text { vs. } \\
\text { placebo }\end{array}$ & $?$ & $?$ & $(-)$ & $(-)$ & $(-)$ & $(-)$ & $?$ \\
\hline
\end{tabular}

MTC: Medullary thyroid cancer; n.d.: Not described; RR-WDTC: RAI-refractory WDTC.
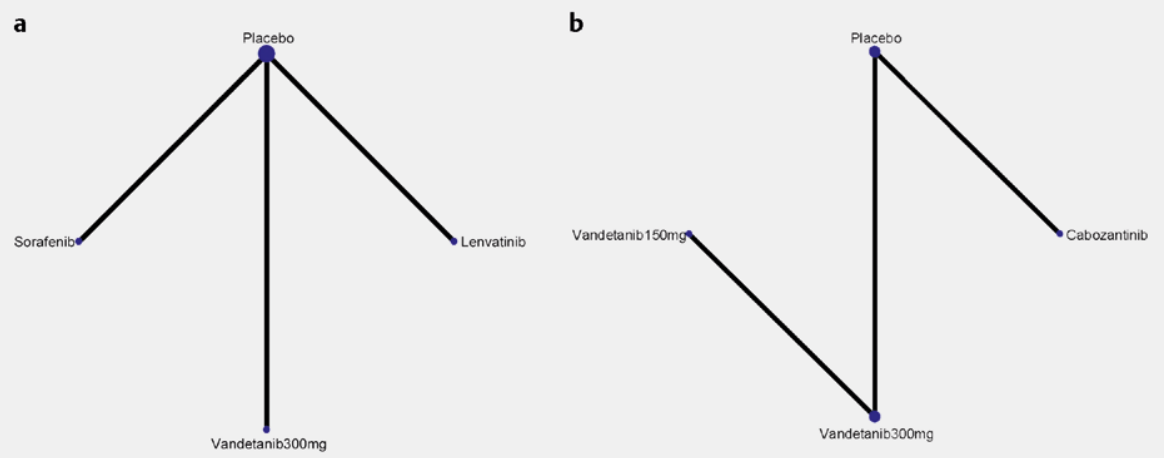

- Supplementary Fig. $2 \mathrm{~S}$ a Network graph of Multikinase Inhibitors in radioiodine-refractory well-differentiated thyroid cancer for disease-control rate, progression-free survival and serious side-effects meta-analyses. b Network graph in medullary thyroid cancer for objective-response rate and serious side-effects meta-analyses. 